\title{
Commentary on Zicari. Expressive Tempo Modifications in Adelina Patti's Recordings: An Integrated Approach
}

\author{
JAAN ROSS[1] \\ Estonian Academy of Music and Theatre
}

\begin{abstract}
In this commentary, statistical and sound signal segmentation methods as applied in Massimo Zicari's "Expressive tempo modifications in Adelina Patti's recordings: An integrated approach" are discussed. A potential method of further analyzing the obtained data is also highlighted.
\end{abstract}

Submitted 2017 January 20; accepted 2017 May 5.

KEYWORDS: music performance, audio recordings, lyrics, melody

THIS paper explores four arias from operas by Mozart and Bellini, performed by Adelina Patti and recorded in 1905 and 1906. It focuses on expressive tempo modifications in particular, with the aim of establishing whether there is a correlation between the words and the temporal structure of the melody. Beat lengths were measured in recorded performances, and simple statistics (mean, standard deviation, mode, and coefficient of variation) applied to measurement results. The paper combines approaches from historical musicology on the one hand, and empirical musicology on the other.

My first comment is related to the use of statistics in this contribution. Internal variability of beat length is expressed by two variables in parallel: the standard deviation in Tables 1 to 4 and the coefficient of variation in Table 5 (which is a part of the discussion section). Since the coefficient of variation is equal to the standard deviation divided by the mean value, no new data are presented in Table 5, as both the mean and standard deviation for the four analyzed arias are given previously in Tables 1 to 4 . Since Tables 1 to 4 are uniform on their content, their formatting might also have been uniform: e.g., the rightmost column in Tables 1,2 , and 4 is indicated as "Mean BL" but in Table 3 as "Mean BL and SD", while in all four cases, values of standard deviation are presented.

Secondly, when presenting data regarding "Voi che sapete", the author writes: "The mean value of the crotchet beat lengths $(\mathrm{N}=137)$ is $1.22 \mathrm{sec}(\mathrm{J}=48)$, which corresponds to the initial beat. The mode value of the beat lengths is also $1.22 \mathrm{sec}(J=48)$, thus suggesting a certain consistency in the chosen tempo." I remain unsure as to whether coinciding values of the mean and the mode can suggest consistency in tempo. Rather, the numerical value of the mode is the same as that of the mean (and median) in a normal distribution, and it may be very different in highly skewed distributions. A performance with a lot of ritardandi and accelerandi can also yield in a normal distribution of beat lengths, as the two compensate for one another. Consistency in tempo may be more reliably estimated using the standard deviation and the coefficient of variation.

My third comment concerns the method used to segment a continuous sound signal into consecutive beats. The author has been concise here as writing: "In order to quantify tempo modifications the inter-onset intervals were measured by tracking each beat onset (crotchet) with a marker in the waveform. When the voice and the piano were not perfectly together the onset in the voice was considered." Providing a waveform with markers is not a straightforward task (see Ross \& Lehiste, 2001, p. 93). For example, consider two consecutive notes connected with a portamento. Where should the marker be placed: at the beginning of a glide, at the end of it, or in the middle? The reader would perhaps have preferred to see more detail here regarding how the segmentation procedure was carried out. The author might have further tried to estimate the accuracy of that procedure, i.e. the temporal resolution.

Finally, there are some data resources which remain unexplored in the paper in its present form. Each aria performed by Patti is represented by two scores (two out of four of them are given in the Appendix). Seeger (1958) has distinguished between two kinds of musical notations, prescriptive and descriptive. Prescriptive notation is usually associated with Western classical music, and understood as the idea of composition written down by the composer as a score. Contrastingly, folk music, for example, is never based 
on score: it can exist in notated form, but these notations are usually not the starting point of the presentation, but have instead been notated after the performance has taken place. Such notations are called descriptive. In the present case, we see both prescriptive (Mozart's and Bellini's scores) and descriptive notations ("as faithful [...] transcription[s] as possible of what can be heard in the audio track") of the same arias. Systematic comparison of these two types of notations of the same musical material would perhaps have strengthened the conclusion made in the paper, that the analysis "challenge[s] the assumption that interpreters trained in the late romantic culture abused the composer's intentions and indulged in tasteless, exaggerated interpretive choices."

\section{END MATTER}

\section{Acknowledgements}

This research has been supported by the Centre of Excellence in Estonian Studies (CEES, European Regional Development Fund) and is related to research project IUT12-1 (Estonian Research Council).

\section{NOTES}

[1] Correspondence can be addressed to: Dr. Jaan Ross, Estonian Academy of Music and Theatre, Tatari 13, 10116 Tallinn, Estonia, e-mail jaan.ross@gmail.com

\section{REFERENCES}

Ross, J., \& Lehiste, I. (2001). The temporal structure of Estonian runic songs. Berlin and New York: Mouton de Gruyter, https://doi.org/10.1515/9783110885996

Seeger, C. (1958). Prescriptive and descriptive music writing. Musical Quarterly, 44, 184-195. https://doi.org/10.1093/mq/XLIV.2.184 